Supporting Information

\title{
On the Growth Mechanism of Transition Metal Dichalcogenide Monolayers: The Role of Self-Seeding Fullerene Nuclei
}

\author{
Jeffrey D. Cain ${ }^{1,2}$, Fengyuan $\mathrm{Shi}^{1,3}$, Jinsong Wu ${ }^{1,3}$, Vinayak P. Dravid ${ }^{1,2,3^{*}}$ \\ ${ }^{I}$ Department of Materials Science and Engineering, Northwestern University, Evanston, Illinois \\ 60208, USA \\ ${ }^{2}$ International Institute for Nanotechnology, Northwestern University, Evanston, Illinois 60208, \\ USA \\ ${ }^{3}$ Northwestern University Atomic and Nanoscale Characterization Experimental (NUANCE) \\ Center, Northwestern University, Evanston, Illinois, 60208, USA \\ v-dravid@northwestern.edu
}




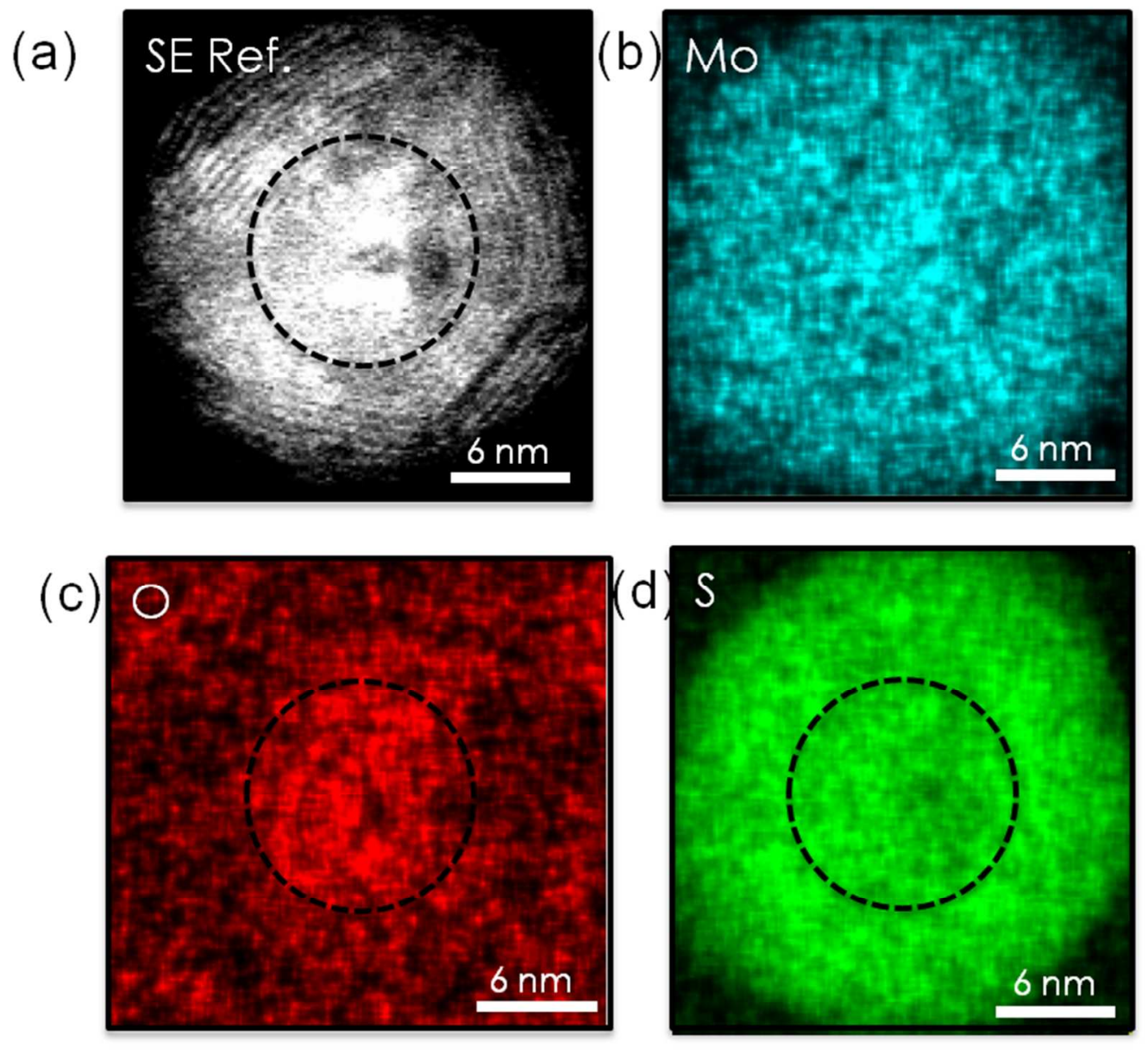

Figure S1. Energy dispersive spectroscopy (EDS) maps of the core shell nucleus structure. (a) Secondary electron reference image of core. (b) EDS map of the molybdenum K series. (c) EDS map of the oxygen K series. (d) EDS map of the sulfur K series 

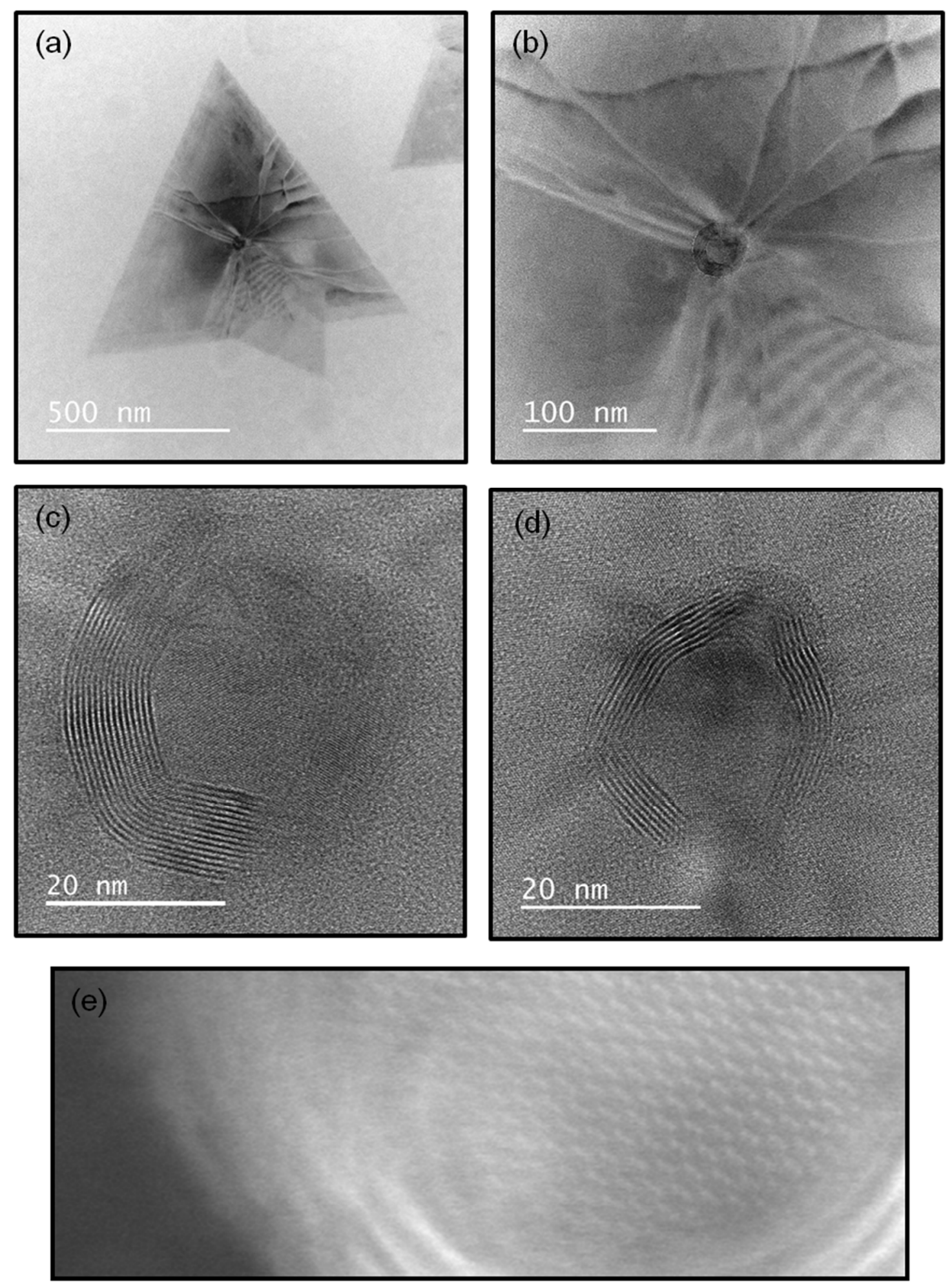

Figure S2. Additional transmission electron microscope images of flakes and nuclei (a) Multilayer $\mathrm{MoS}_{2}-\mathrm{MoSe}_{2}$ sheet (b) Low-magnification image of nucleus (c) and (d) Highmagnification images of nuclei (e) HREM image of nucleus core. 


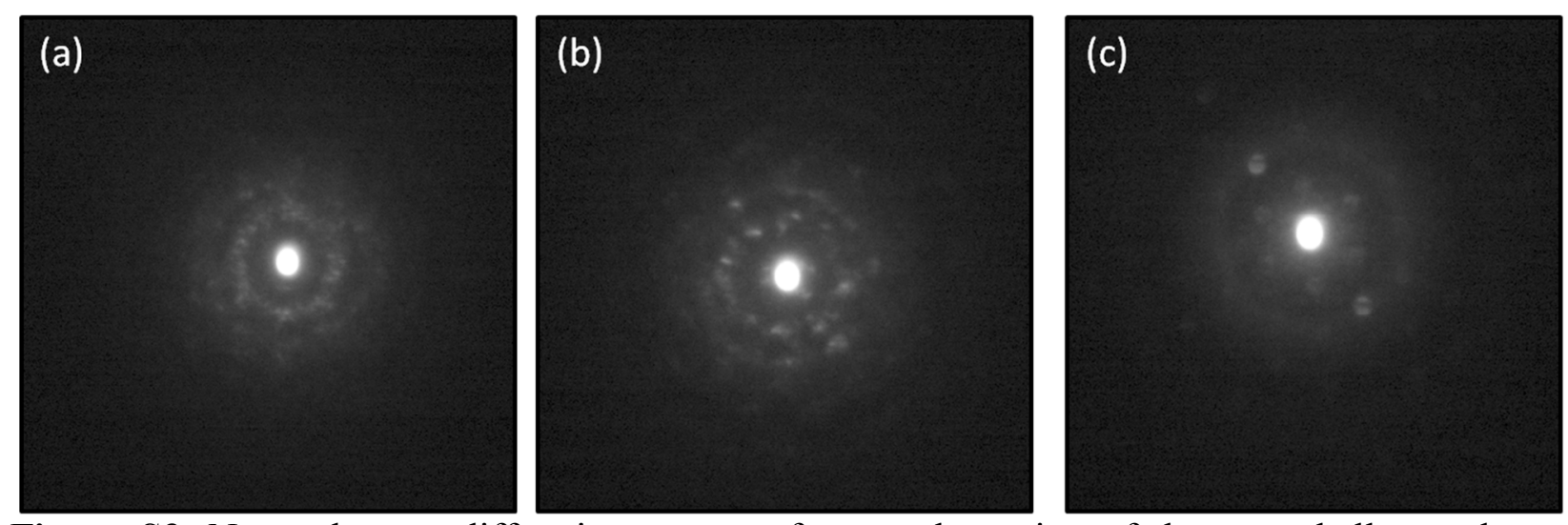

Figure S3. Nano electron diffraction patterns from each section of the core-shell-monolayer structure. (a) Nucleus center (b) Nucleus Edge (c) Monolayer film

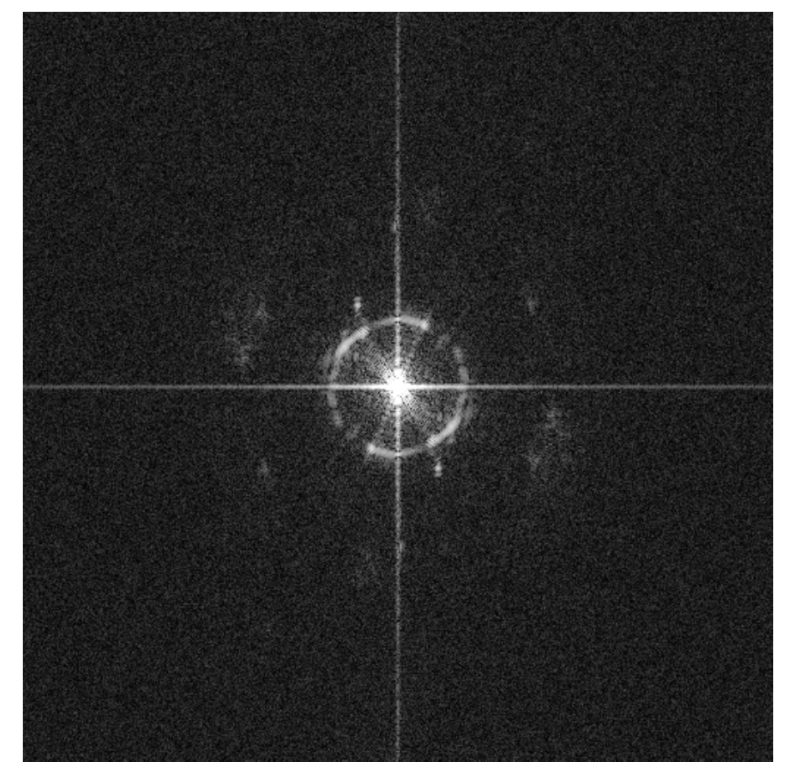

Figure S4. Fast Fourier Transform of the false colored image shown in Figure 2. 